\section{Die Gewinnung und Entfernung von Naturstoffen durch "Aufschließen“.}

Von Privatdozent Dr. Viktor Grafe, Wien.

Die unaufhörlich sich steigernden Kulturbedürfnisse der Menschheit haben das Bestreben erzeugt, künstlich mehr van jenen Produkten zu schaffen, mit denen die Natur uns allzu stiefmütterlich bedacht hat. Nun ist aber die Natur eine Meisterin, die sich ihre Geheimnisse nicht so ohne weiteres ablauschen läßt und die ihre Künste eifersüchtig hütet. Darum bedeutet es immer einen Markstein in dem heißen Wettstreit zwischen Natur und Mensch um die Vorherrschaft auf.Erden, wenn der Natur wieder eines ihrer Geheimnisse entrissen worden ist. Haben wir aber einmal die Natur erreicht, dann ist sie auch schon übertroffen, denn im Laboratorium des Lebens kommt es selten zur Bildung eines einheitlichen Stoffes, der ohne weiteres direkt für den Mensehen verwertbar wäre. Vielmehr müssen meist erst umständliche Reinigungsverfahren die unwesentlichen und störenden Verunreinigungen entfernen, während die zielbewußte Arbeit des Chemikers den Körper, dessen Aufbau einmal erkannt ist, gleich in idealer Vollkommenheit zu erzeugen vermag. Solche Großtaten menschlicher Kombinationskraft sind die Synthese des Krappfarbstoffes, des Indigo, des Veilchenduftstoffes Jonon, lauter Produkte, die wir heute genau der Natur nachzugestalten vermögen, wobei wir aber unsere Lehrmeisterin an Vollendung übertreffen, abgesehen davon, daß unsere Ausgangsmaterialien und damit das Produkt der Synthese wohlfeiler sind als uns das Naturprodukt zu stehen käme.

In vielen Fällen, in denen wir durchaus auf die Naturprodukte angewiesen sind, werden diese von der Natur nicht in einer für die Gewinnung geeigneten Form, in den meisten Fällen nicht ,frei“" sondern in chemischen Verbindungen geboten, aus denen die wirksamen, wertvollen Stoffe erst durch besondere Verfahren in Freiheit gesetzt werden müssen. Hier sind es im wesentlichen zwei Wege, auf denen der Mensch sein Ziel erreicht. Der erste ist ganz eigenartig, indem der Mensch die Natur selbst zwingt, die ihm genehmen Stoffe zu schaffen, die im normalen Ablauf der Dinge gar nicht gebildet würden, daß er durch sein Eingreifen den Ablauf des Naturprozesses so dirigiert, daß als Resultat der gewünschte Körper erscheint, der dann also im Stoffwechsel eines Organismus entstanden ist, aber erst nachdem der Anstoß zu seiner Entstehung von Menschenhand gegeben wurde. Dieses Verfahren - das Fermentieren - beruht auf der Schaffung von Bedingungen, unter denen die fermentative Spaltung der komplexen Verbindung, in welcher der fragliche Stoff vorliegt, gefördert wird. Auf diese Weise kann die natürliche Entstehung von Riechstoffen, von Alkaloiden usw. dirigiert werden. Der andere Weg - das Aufschließen ist ein rein chemischer, er beruht auf der Tatsache, daß ,schwächere" Verbindungen durch "stärkere" aus Komplexen mit anderen Stoffen ausgetrieben werden. Dieser letztere Weg findet besonders dann
Anwendung, wenn es sich darum handelt, irgendeinen bestimmten Stoff aus dem Naturprodukt zu entfernen, ohne daß der andere mit ihm im Komplex befindliche Stoff mitentfernt werden soll, wenn es sich also gewissermaßen um eine Isolierung handelt. Fin Beispiel wird das deutlicher machen. Im Tabakblatt ist der größte Teil der Nikotinbase an organische Säuren (Äpfelsäure, Zitronensäure usw.) gebunden, in der Kaffeebohne ein großer Teil des Koffeins an Chlorogensäure. Wollte man beim Entnikotinisieren oder Dekoffeinisieren direkt mit Extraktionsmitteln vorgehen, so würde man, abgesehen davon, daß die Extraktion nur sehr unvollkommen verliefe, mit dem Nikotin, bzw. Koffeïn auch die mit ihnen in Verbindung stehenden Säuren entfernen, die z. T. ein gut Teil der Wirkung der betreffenden Genußmittel veranlassen. Wird aber vorher durch Aufschließen für die Lösung der Verbindung Sorge getragen, dann wird die Extraktion des freigelegten Nikotins oder Koffeins in grofem Betrage erfolgen können und nur diese betreffen. SchlieBlich versteht man unter Aufschließen noch ein anderes Verfahren, das ausschließlich Nahrungsmittel betrifft, nämlich die Lockerung des Molekulargefüges durch chemische oder physikalische Eingriffe, wodurch der betreffende Nahrungsstoff für die Verdauungssäfte des menschlichen Körpers leichter zugänglich gemacht wird.

Wenn wir zunächst einige Fälle von Fermentation betrachten wollen, so sei zunächst daran erinnert, daß sich der bekannte blaue Farbstoff Indigo niemals in der lebenden Pflanze als soleher fertig. gebildet vorfindet, sondern nur dessen ungefärbte Muttersubstanz, das Indikan. Zur Gewinnung des blauen Farbstoffes müssen die abgesehnittenen Pflanzen zunächst abgetötet werden. Man weicht sie einige Stunden in lauwarmem Wasser ein, wodureh das Indikan aus den Blättern herausgelöst wird und gleichzeitig ein in dem abgestorbenen Protoplasma befindliches Ferment, das die Spaltung des Indikans in Indigweiß und Zucker vollzieht. Am Ende der Extraktion, nach etwa zehn Stunden, ist die Flüssigkeit nicht auffallend gefärbt; aus dem Extraktionsbassin ergießt sie sich - so schildert Molisch den Proze B aus eigener Anschauung - in mächtigem Strom in ein anderes Bassin und schon nach wenigen Minuten bildet sich himmelblauer Schaum, ein Rührwerk mit hölzernen Schaufeln setzt sich in energische Bewegung, der Extrakt wird ,,geschlagen“, d. h. ordentlich mit Luft durchmischt, und binnen zwei Stunden ist das gesamte Indigweiß zu Indigblau oxydiert, wobei durch Zusatz von Ätzkalk die Indigobildung befördert wird. Ebenso wie durch ein spaltendes Ferment aus dem Chromogen Indikan der Farbstoff Indigo gebildet wird, so zerfällt die goldgelbe Ruberythrinsäure der Krappwurzel nach dem Abtöten in Zucker und den roten Alizarinfarbstoff. Es ist überhaupt eine charakteristische, industriell noch kaum gewürdigte Erscheinung, daß nach dem Tode des Protoplasmas, in der sog. Autolyse, oder auch nur während des durch Narkose hervorgerufenen lethargischen Zustandes, kurz, wenn dessen die Enzymarbeit regulierendes Wirken sus- 
pendiert oder zerstört ist, die Fermente vornehmlich in der Richtung des Abbaues tätig sind, also z. B. aus Glukosiden wie Indikan oder Ruberythrinsäure die Komponenten Zucker und Farbstoff abspalten, aus hochmolekularen Kohlehydraten die einfachen Zuckerarten abbauen. So habe ich in stärkehaltigen Knollen und Keimpflanzen, nachdem sie kurze Zeit in Chloroform-, Ather-, Azetylennarkose gehalten worden waren, Anhäufung von Zucker, in eiweilhaltigen solche von Aminosäuren beobachten können. Es ist $j a$ auch wahrscheinlich, daB dem durch Äther hervorgerufenen Frühtreiben von Pflanzen derselbe Prozeb zugrunde liegt, indem die gebildeten einfachen Kohlehydrate ebenso als Baustoffe Verwendung finden wie das normalerweise bei deren Frühlingsmobilisierung statt hat. Auch beim Tierkörper vollzieht sich in der Narkose etwas ahnliches, auch hier wird ein UbersehuB von Zucker durch Abbau von Glykogen gebildet und im Harn ausgeschieden - die "Narkosediabetes".

Das Vanillin, der bekannte intensive Duftstoff der Vanilleschote, ist nirgends im Pflanzenreich fertig gebildet vorhanden. Die frischen, reifen Vanilleschoten sind nahezu geruchlos und enthalten von dem aromatischen Stoff kaum nennenswerte Mengen. Erst durch eine besondere Art der Zubereitung der Früchte gelingt es, das Vanillin selbst freizumachen. Es sind da zwei Arten für den guten Ertrag der Ernte maßgebend, das trockene Verfahren, wie es in Mexiko, dem Zentrum der Vanillegewinnung geübt wird und das HeiBwasserverfahren. Bei der mexikanischen Trocknung werden die Früchte auf einem hölzernen Gitterroste flach ausgebreitet und zunächst zum Welken gebracht, dann auf schwarzen Wolldecken liegend, der Sonnenglut ausgesetzt und schließlich schnell in vorher angewärnte Kasten verteilt, die Wolldecken über ihnen zusammengeschlagen und nun ein regelrechter SchwitzprozeB eingeleitet, der 16 bis 22 Stunden dauert, wonach die Frucht jene dunkelbraune Farbe angenommen hat, mit der wir das Handelsprodukt ausgestattet sehen. Schließlich kommt der wichtigste ProzeB, die Fermentwirkung, zu welcher die Schoten einen Monat an der Sonne oder im Backofen liegen müssen, um zu „kristallisieren“, d. h. sich mit einer Schichte der glänzend weißen Vanillinkristalle zu überziehen, welche jetzt erst das Aroma der Früchte hervorrufen. Stat der Sonne oder des Ofens verwendet das HeiBwasserverfahren siedendes Wasser, in das die Schoten mehrmals für einige Sekunden getaucht werden, um dann erst regelrecht zu schwitzen. Der Vanillesaft birgt neben der geruchlosen Muttersubstanz des Vanillins ein Ferment, das Fmulsin, welches die schwachriechende Muttersubstanz des Vanillins, das Glukovanillin, in Zucker und Vanillin zerlegt. Das Glukovanillin mag seinerseits durch Oxydation aus Koniferin entstehen, so genannt, weil es sich auch im Rindensaft unserer Koniferen findet; aus ihm kann das Vanillin auch künstlich dargestellt werden. Jedem ist bekannt, daß ein frisches Fichtenbrettchen, an den geheizten Ofen gestellt, einen mehr oder weniger intensiven Vanillegeruch ansströmt. Das kommt daher, daß teils kleine Mengen Vanillin, die aus dem Koniferin entstanden sind, sich als ständige Begleiter dex Holzsubstanz finden, teils durch die Ofenwärme eine Spaltung des Glukovanillins in Zucker und Vanillin stattfindet, genau so wie beim SchwitzprozeB der Vanilleschoten. In der Vanillefrucht aber entsteht durch Wirkung des Emulsins noch nicht Vanillin, sondern erst eine Vorstufe desselben, der Koniferylalkohol, während das Vanillin selbst ein Aldehyd ist. Da Aldehyde im allgemeinen stärker riechen als die Alkohole, aus denen sie durch Oxydation entstehen, so ist wohl auch schon durch Entstehung des Koniferylalkohols ein gewisses Aroma gegeben, aber erst durch dessen Oxydation zum Aldehyd tritt das typische Vanillearoma hervor. Diese notwendige Oxydation nun bewirkt wieder ein anderes Ferment, eine Oxydase, die sich neben Fmulsin in der Vanilleschote findet. So ist es wohl auch im Holzbrettchen, dessen Geruch zuerst schwach ist und erst bei längerem Trocknen intensiver wird, denn die Wirkung der Oxydase wird erst durch höhere Temperaturen zu voller Geltung gebracht. Diese Produkte treten also im Leben gar nicht in Erscheinung, in der Natur ist das Vanillin gar nicht als solches vorhanden, d. h. es ist kein Endprodukt eines Stoffwechselprozesses, sondern ein Zwischenprodukt; die spaltenden und oxydierenden Fermente, die bei der Erzeugung dieser für den Menschen wertvollen Produkte beteiligt sind, finden in lebenden Pflanzenkörpern eben nicht die für ihre Wirksamkeit, notwendigen Arbeitsbedingungen, kommen also im natürlichen Laufe der Dinge gar nicht dazu, diese Produkte zu erzeugen, sondern erst, nachdem ihnen die notwendigen Wirkungsverhältnisse künstlich zur Verfügung gestellt worden sind.

Auch die Blätter des frischen Patschulikrautes sind ohne jeden Duft, das wohlriechende Patschuliöl bildet sich in ihnen erst durch eine Art Gärung, der die getrockneten Blätter unterworfen werden; in Indien destilliert man darum die getrocknete Pflanze mit Wasserdampf, während andere Blütenblätter und Laubblätter, in denen der Duftstoff bereits fertig gebildet steckt, in frischem Zustande verarbeitet werden müssen. Zur Ausfuhr nach Europa werden die Patschuliblätter halbtrocken verpackt und absolvieren die notwendige Fermentation im Schiffsraum. Der Duftstoff des Steinklees, des Waldmeisters, des getrockneten Grases, das Kumarin, kommt in der lebenden Pflanze ebenfalls nicht, oder nur in geringer Menge oder schließlich an andere Substanzen gebunden vor. Gras und Steinklee duften wohl auch in lebendem Zustande jedermann weib aber, dab ihr Heu einen viel stärkeren Duft ausströmt, indem eben dann das Kumarin gebildet oder aus seiner Verbindung abgespalten wird. Auch in der brasilianischen Tonkabohne, die in der Parfümerie wegen ihres Duftstoffes zur Parfümierung der künstlichen ,Weichselhölzer" und zur Bereitung der Maitrankessenz reichliche Anwendung findet, kommt das Kumarin nicht als solches vor, sondern die aus der Frucht herausgelösten Samen, die Tonkabohnen, müssen erst eine absonderliche Prozedur durchmachen. Man 
schüttet sie in große Fässer, füllt dann das Faß mit Rum und bedeckt es mit Sackleinwand; nach 24 Stunden zieht man den Rum wieder $a b$ und trocknet die Bohnen an der Luft. Sie sind dann fast schwarz und aufgeblasen, und went sie trocken geworden sind, erscheinen sie an der Oberfläche mit weiBen glänzenden Kumarinkristallen bedeckt, denen sie thren wunderbaren Duft verdanken.

Der wertvolle Inhaltstoff kommt auch in unseren verbreitetsten GenuBmitteln, dem Kaffee, Tee, Kakao, Tabak, nicht in freier Form oder in ungenügender Menge vor, so daB auch bei ihnen die Erntebereitung zum großen Teil in einer aufschließenden Fermentation besteht, wobei durch Fermente das wirksame Alkaloid aus einer inaktiven Verbindung abgespalten wird. Das Koffein des Tees z. B. ist in de11 Teeblätern in Form eines Glukosids an Zucker gebunden, vorhanden, das erst durch Fermentwirkung gespalten werden muB. Durch die fermentative Spaltung des Koffeinglukosids entsteht Koffein und Teerot, dem der schwarze Tee seine Farbe verdankt. Auch das Teearoma wird bei der Fermentierung erzeugt. Ftwas ähnliches dürfen wir beim Tabak annehmen; das Nikotin ist im frischen Tabakblatt an Zitronensäure und Äpfelsäure gebunden; auch hier muB die Fermentierung eingreifen, die dem Tabak seinen charakteristischen Geruch und die dem Raucher erwünschten Eigenschaften verleiht. Die Kakaofrucht, das Ausgangsmaterial für die Schokolade, ist zunächst bitter und herb; erst durch eine mehrere Tage währende Formentierung wird der angenehme milde Geschmack und das feine Aroma erzeugt. Sehr verschieden von diesen Fermentwirkungen ist die Gärung, welche die Kaffeebohnen bei der Erntebereitung durchmachen; sie hat hier nur den äuberlichen Zweck, das Abfaulen der anhängenden Fruchtschalenreste zu bewirken. Das, was die Fermentierung bei Tee und Kakao vollzieht, die Spaltung der zusammengesetzten Muttersubstanzen wird beim Kaffee z. T. durch das Rösten bewirkt. Wir werden später hören, was daraus für den Proze B des Entkoffeivisierens folgt. Bei manchen Genumitteln erfolgt die Fermentation crst beim Genusse selbst, so beim Kolakauen durch den Speichel des Kauenden. Unbewußt haben die Neuseeländer diese wissenschaftliche Methode auch beim Genusse der Kawa gefunden. Das ist die Wurzel einer Pfefferart, Piper methysticum, die auf den Südseeinseln rorkommt. Die Wurzel wird abgeschabt, geschnitten und zu Brei zerkaut. Durch das Ptyalin des Speichels geht ein AufschlieBprozeB vor sich, der das Narkotikum erst frei macht. Das Ptyalin besorgt bekanntlich ebenso wie die Diastase des Malzes, auch eine Verzuckerung der Stärke und tatsächlich wird ron den Indianem in Guyana der Speichel auch benutzt, um stärkehaltige Materialien zu verarbeiten, indem sie dieselben kauen, das Gekaute ausspucken und den mit Wasser verdünnten, nunmehr zuckerhaltigen Brei vergären lassen. Sehr merkwürdig ist folgendes Aufschließverfahren. In den Laubblättern der Apfel, Birnen, Himberen, des Weines sind die Geruchstoffe latent, welche das Aroma der betreffenden Früchte ausmachen, in duftlosen chemischen Verbindungen enthalten. Wenn man nun einen Brei aus den betreffenden 'Laubblättern herstellt und eine Aufschwemmung mit Zuckerwasser bereitet, gelingt es beim Vergären des dünnflüssigen Breies durch gewöhnliche Weinhefe ein alkoholisches Getränk zu gewinnen, welches den Duftstoff der betreffenden Frucht in desto ausgeprägterem Maße zeigt, je näher der Zeit der Fruchtreife die Blätter der Pflanze entnommen werden. Die Hefe bewirkt durch ein Enzym hier das Aufschlieben, und es zeigt sich, dab der charakteristische Duftstoff nicht nur in den Früchten enthalten ist, sondern offenbar in den assimilierenden Laubblättern gebildet wird, in den Früchten aber aus einer komplexen, duftlosen Verbindung erst in Freiheit gesetzt werden kann, ein Vorgang, der meistens als ein im wesentlichen lichtchemischor erkannt worden ist; dem entspricht auch die Erfahrung, daB die Früchte um so aromatischer ausfallen, je lichtreicher der Sommer war. Durch das genannte Verfahren gelingt es also, das Fruchtaroma ohne Früchte zu erzeugen. Einen Fermentationsprozeb macht auch der südamerikanische Paraguayteo oder Maté durch, die Blätter und Zweige mehrerer Arten der Gattung llex aus der Familie der Aquifoliaceen. Es sind Stechapfelarten, die im Gegensatz zu den unsrigen neben einem koffeinartigen Alkaloid noch ein ätherisches öl enthalten, das nicht nur für Aroma, sondern auch für die Wirkung wesentlich zu sein scheint. Beide werden erst bei der Fermentation entwickelt. Die Zweige wurden bei dem von den Eingeborenen befolgten Verfahren abgeschnitten, über lebhaftem Feuer getrocknet, wobei es häufig vorkam, daß dem GenuBmittel ein unerwünschter Rauchgeschmack gegeben wurde. In den modernen, von Deutsehen begründeten Maté-Betrieben wird das jetzt vollkommen vermieden und ein sorgfaltig behandelter Maté nach Europa gebracht. Die Behandlung über dem Feuer wird mehrere Male wiederholt und in der Zwischenzeit machen die Zweige die Fermentation durch, das fertige GenuBmittel wird in derselben Weise zubereitct wie unser Teo. Der Geschmack dieses für Europa neuen GenuBmittels, das in seiner südamerikanischen Heimat schon lange bekannt und leidenschaftlich geschätzt ist, weicht von dem unserer gewohnten GenuBmittel einigermaßen ab, ist aber durch das Vorhandensein von Gerbstoffen und dem genannten aromatisehen ol kräftig und herzhaft und sagt nach kurzer Gewöhnung auch dem europäischen Gaumen ungemein zu. Auch als Extrakt oder als Tabak kann Matë genossen werden. Einer Arbeit von Dr. P. Benignus, welcher im Heimatlande den Maté gründlich kennen gelernt hat, entnahm ich die Bemerkung, daß Maté modizinisch verwertbar ist, keine nervenaufregende, sondern nervenanregende Wirkung zeigt, füx Kranke, Rekonvaleszenten, Kinder wegen seiner absoluten physiologischen Harmlosigkeit geeignet ist und als vorzüglicher Energieentwickler für den körperlichen und geistigen Arbeiter bezeichnet werden muB, daß Maté überdies bakterientötend wirkt und in wunderbarer Weise den Durst stillt und das Hungergefühl niederhält. Durch seine große Wohlfeilheit und die Möglichkeit, 
dasselbe Quantum Maté zu mehreren Aufgüssen verwenden zu können, ist er besonders geeignet, einer größeren Menge von Arbeitern oder Soldaten angenehme und anregende Erfrischung zu bieten. In neuester Zeit ist aber aus Maté auch ein Genuß. mittel geschaffen worden, das dem verwöhntesten Gaumen zusagt und bestimmt ist, von jenen Kreisen bevorzugt zu werden, welche gegenüber den alkoholischen Tischgetränken eine Abwechslung suchen oder den Alkohol vermeiden wollen, ohne die Nervenanregung zu entbehren. Es handelte sich auch hier wieder darum, die wertvollen Matébestandteile aus Verbindungen zu lösen, sie löslich und nutzbar zu machen. Ein sehr einfacher AufschließprozeB, dessen Beschreibung mich hier zu weit führen würde, machte das möglich, und so gelingt es, aus Matéxtrakt die verschiedensten GenuBmittel zu schaffen, anregende Nährpräparate, Getränke wie die sog. Bronten, welche mit den alkoholfreien Getränken nicht nur deshalb.konkurrieren können, weil sie im Gegensatz zu diesen reine Naturprodukte sind, sondern auch, weil sie die erwünschte Anregung nicht entbehren lassen; ferner die aufgeschlossenen Aufgußpräparate selbst wie Rio-Matte und Ete.

Eine sehr wichtige Rolle spielt das Aufschließen bei der Entgiftung unserer GenuBmittel. Wem allen Menschen das Bedürfnis nach Nervenreiz angeboren $\mathrm{zu}$ sein scheint und sich auf bestimmter Kulturstufe der Völker von selbst vordrängt, so verläuft doch die Freude an der Nervenanregung im Dasein der Menschheit in Form einer Kurve, deren aufsteigender Ast, an einem bestimmten Punkte der Entwicklung beginnend, bis zu einem Gipfelpunkte zicht, von dem aus der absteigende Ast, dic Reaktion gegen das Genußmittel, anhebt. Freilich bis wieder zu Null absinken wird die Kurve nicht, wenigstens könnten wir uns nicht vorstellen, was geschähe, wenn alle Genußmittel wieder aus unserem Leben verschwänden. Immerhin aber hat die Reaktion eingesetzt, der rivilisierte Mensch besinnt sich auf dic Schäden, welche das nervenanregende Gift für die übrigen Lebensfunktionen mit sich bringt und daß die sprichwörtliche „Nervosität" des Kulturmenschen zum großen Teil den GenuBmittelgiften zuzuschroiben ist, deren Verbrauch mit der Tast des modernen Frwerbslebens Hand in Hand mit der Gewöhnung an das Gift steigt, so dal immer weitere Kreise der dauernden Schädigung verfallen. Ein charakteristischer Indikator für die immer wachsende Empfindlichkeit des Kulturmensehen ist wohl darin zu sehen, daß während früher im wesentlichen nur das allgemeinste und stärkste Genußmittelgift, der Alkohol, leidenschaftlich bekämpft wurde und man beispielsweise Koffein gerade an Stelle von Alkohol gesetzt wissen wollte, man sich heute auch schon von dem Glauben an dio Marmlosigkeit dieses Alkaloids emanzipiert und es möglichst zu rermeiden sucht, wenigstens dort, wo die Widerstandskraft des Organismus durch Krankheit geschwächt ist. Die Antialkaloidbewegung sucht nun dem GenuBmittel das Alkaloid bis zur physiologischen Harmlosigkeit zu entziehen, wobei es jedoch ein wichtiges Problem ist, nicht auch aroma- und geschmackgebende Stoffe mit dem Giftstoff $z u$ entfernen. Nicht überall macht es ja die Natur dem Menschen so leicht wie bei den alkoholischen Getränken, bei denen durch gelinde $\mathbf{E r}$ hitzung der Alkohol ausgetrieben werden kann oder wie bei den Fruchtsäften, die durch Pasteurisieren vor unerwünschter Gärung bewahrt werden können. Wenn man nämlich dem Tabak oder Kaffee das wirksame Alkaloid zu entziehen versucht, macht man die Frfahrung, daß dies nicht so ohne weiteres möglich ist, denn hier sind die Alkaloide so fest an die organischen Säuren oder die Gerbstoffe gebunden, daß die gebräuchlichen Lösungsmittel nur gerade jenen Teil des Alkaloids entfernen, der gerade frei vorliegt, nicht aber die Hauptmenge, welche chemisch gebunden ist. Dazu kommt noch, dab, wie bereits erwähnt, die genannten organischen Komplexe für Geruch und Geschmack sehr wesentlich mitverantwortlich sind, so daß mit ihrer Entfernung das GenuBmittel ganz wertlos gemacht werden müßte. Hier setzte das Aufschließverfahren ein. K. Wimmer, Bremen, D.R.P. Nr. 124875, kam zuerst auf den Gedanken, das Rohmaterial einem Aufschließprozeß zu unterwerfen. In der Kaffeebohne ist der gesamte Koffeingehalt in Form der Verbindung mit der erst kürzlich durch Gorter näher bekannt gewordenen Ohlorogensäure vorhanden. Das Aufschließverfahren löst zunächst diese Verbindung zum größten Teil, es besteht in einer Behandlung der Bohnen mit gespanntem Wasserdampf; das in Freiheit gesetzte Koffein kann nummehr durch reinstes Benzol zum gröBten Teil extrahiert werden, welches selbst nachher durch Wasserdampf wieder vollkommen weggeblasen wird. Vom ursprünglichen Gehalt von $1,4 \%$ findet eine Verminderung bis auf $0,1 \%$, eine physiologisch ganz belanglose Quantität, statt. Das Benzol reinigt gleichzeitig die Bohne grundlich von anhaftendem Fett und Wachs, welche, namentlich bei unsorgfältigem Rösten, Valeriansäure und brenzliche Produkte entstehen lassen können. Aber die Wasserdampfbehandlung leistet noch mehr, sie lockert die Zellen auf, welche bei mikroskopischer Betrachtung von Kaffeebohnenschnitten mit stark verdickten Zellwänden versehen erscheinen und löst einen Teil dieser Substanzen, der Henizellulosen, die für den werdenden Kaffeebaum beim Keimen des Samens die Reservestoffe bilden, auf. Diese sind aber auch gleichzeitig die Muttersubstanzen von gewissen Röststoffen, welche infolge dieses Umstandes im entkoffeinisierten Kaffee wesentlich an Quantität und Zusammensetzung herabgemindert sind. Die beim Rösten der Kaffeebohne unter deren Braunfärbung entstehenden aromatischen Substanzen, welche in den wässerigen Auszug übergehend, das spezifische Aroma des Kaffeegetränkes ausmachen, werden in ihrer Gesamtheit Kaffeol genannt und in neuerer Zeit von manehen Medizinern neben oder sogar vor dem Koffein für die physiologische Wirkung des Kaffees verantwortlich gemacht. Es ist tatsächlich auffallend, daß der Tee, obgleich er erheblich mehr Koffein enthält als der Kaffee und im allgemeinen bei den in Haushalten gebräuchlichen Aufgüssen der Koffeingehalt einer Tasse Tee 
nahezu derselbe ist wie der eine Tasse Kaffee, bekanntermaBen physiologiseh wesentlich harmloser ist als der Kaffee. So wird z. B. von Harnack, Halle, die Wirkung gewisser Röstprodukte dafür verantwortlich gemacht, die zum großen Teil aus den Gerbstoffen, dem der Bohne anhaftenden Fett und Wachs usw. entstehen sollen. Von J. Thum wurde ein Verfahren angegeben (D. R.P. Nr. 382 238), welehes die Muttersubstanzen dieser Röstprodukte durch einfaches Bürsten der Kaffeebohnen mit lauwarmem Wasser entfernen soll, wobei angeblich gleichzeitig ein relativ erhebliches Quantum Koffein mitentfernt wird. So gewaschener Kaffee soll dann tatsächlich die unerwünschten Röststoffe vermissen lassen. Damit wäre ein höchst einfaches Aufschließverfahren gegeben, aber die Prüfung des Thumschen Verfahrens durch Harnack, welcher di Abwesenheit der schädlichen Röstprodukte hauptsächlich nach der Methode der Tropfengewichtsbestimmung darzutun versucht, und die Bestätigung der erzielten Vorteile sind nicht durchaus überzeugend. Angeregt durch eine Bemerkung in Molisch' Histochemie, wo dieser Forscher es als wichtige Aufgabe bezeichnet, die Aufmerksamkeit darauf zu richten, wo denn die sogenannten wirksamen Stoffe der GenuBmittel ihren Sitz haben, unternahm ich es, die Herkunft der wichtigsten Kaffeeröststoffe zu untersuchen. Uber die Zusammensetzung des Kaffeols sind wir durch eine höchst wertvolle, auf Veranlassung Harnacks durchgeführte Untersuchung von E. Erdmann ziemlich genau unterrichtet, wir wissen, daß ca. $50 \%$ von Furfuralkohol gebildet werden, daneben ist Valeriansäure, Essigsäure und eine stickstoffhaltige aromagebende Substanz vorhanden. Der Furfuralkohol ist zugleich nach $\mathrm{Erd}$ mann physiologisch wirksam. Ich habe nun gefunden, daß durch den oben erwähnten Waschprozeß, die Kaffeolbestandteile kaum verändert werden, nur die Valeriansäure zeigt eine Verminderung. So sehr der Anblick des sich alsbald grünlich-schwarz färbenden, fettigen Waschwassers zu dem Glauben verleiten könnte, es seien hier erhebliche Veränderungen in der Zusammensetzung der Bohne vor sich gegangen, so wenig trifft das tatsächlich $z u$, die entfernten Fett- und Wachsanteile nehmen eben an der Bildung des eigentlichen, in den Auszug übergehenden Kaffeols kaum teil. Beim Entkoffeinisieren verhält es sich anders. Hier findet zunächst eine Auflockerung des Zellgefüges durch die Behandlung mit gespanntern Wasserdampf statt, die Hemizellulosen der verdickten Zellwände werden zerstört und die Bindung von Chlorogensäure und Koffein gelöst. Die nachfolgende Extraktion mit dem fettlösenden Benzol zieht dann nicht nur das freigewordene Koffein aus, sondern reinigt auch die Bohne gründlich von Fett und Wachs. Tatsächlich ergibt solcher, der Entkoffeinisierung unterzogen gewesene Kaffee nicht nur die schon vorerwähnte beträchtliche Verminderung an Koffein, welches für sich gewonnen wird, sondern auch eine auffallende Verringerung des Anteils an Furfuralkohol im Kaffeol, so daßs wohl der Schlul gerechtfertigt ist, daß dieser zum großen Teil aus den Zellwandverdickungen stammt. Die abfallende, sog. Kaffee- schlaufe, welche große Mengen Kaffeewachs enthält, zeigt, wie weit auch nach dieser Richtung das Benzol extrahierend wirkt.

Ahnlich dem koffeinfreien Kaffee wird neuerdings auch koffeinfreier Tee (D.R.P. Nr. 196 885) erzeugt. Obzwar ja schon durch die Erntebereitung der größte Teil des Koffeins in Freiheit gesetzt ist, vervollständigt man diesen ProzeB durch Aufschlielen mittels Säuren oder Alkalien, wobei man vorher für die Extraktion der aromagebenden Stoffe Sorge trägt. Wenn dann das Koffein durch geeignete Lösungsmittel entfernt ist, werden die genannten Stoffe wieder zugefügt. Es braucht nicht betont zu werden, daß durch diese vielfachen chemischen Manipulationen die Qualität des Materials nicht verbessert werden dürfte, wie ja überhaupt beim Tee ein Entkoffeinisieren weniger geboten erscheint als beim Kaffee.

Das Nikotin ist in den Tabakblättern kaum in freier Form vorhanden, sondern als Salz verschiedener organischer Säuren, besonders Äpfelsäure, Zitronensäure, Oxalsäure usw., welche neben den Tabakharzen jedenfalls von wesentlicher Bedeutung für den Geschmack des Genußmittels sind. Darauf muß natürlich bei der Entgiftung des Tabaks besonders Rücksicht genommen werden. Ein Auslaugen der Tabakblätter mit Wasser, welches bei gar zu nikotinreichen Blättern vorgenommen werden muß, weil z. B. ungelaugte Virginia- oder Kentuekytabake kein Mensch rauchen könnte, entfernt natïrlich nicht nur das Nikotin, sondern auch die wertvollen organischen Säuren und z. T. die Tabakharze. Häufig werden dann die Blätter mit aromatischen, die Geruchs- und Geschmacksnerven stark beeinflussenden Stoffen imprägniert, sauciert. Selbst das Besprengen der Blätter mit Wasser oder deren kurzes Eintauchen, um sie geschmeidiger zu machen, kann, ohne die nötige Sorgfalt ausgeführt, schon wertvolle Inhaltstoffe entfernen. Auf dem Gebiete der Tabakentgiftung hat der Erfindergeist eine anerkennenswerte Fruchtbarkeit gezeigt, freilich ohne daß ihm durchaus befriedigender Erfolg beschieden gewesen wäre, denn alle Nikotinlösungsmittel, mit denen man den Tabak behandelt, versagen, da ja der größte Teil des Alkaloids nicht frei, sondern gebunden vorliegt. Man hat versucht, Ozon, Wasserstoffsuperoxyd, ja sogar den elektrischen Strom die Entgiftungsarbeit vornehmen $\mathrm{zu}$ lassen. Laugt man den Tabak aus, so werden auch wertvolle Bestandteile entfernt, das Produkt strohig und qualitätsarm. Die rein meehanischen Mittel zur Entfernung des Nikotins durch Filter von Wolle oder anderes Material, sind so gut wie wertlos, selbst wenn sie mit verschiedenen Stoffen imprägniert sind, welche das Gift „,binden" sollen, denn die Schnelligkeit des Durchzugs macht solche Bindungen illusorisch, die sich bildenden teerartigen Rauchprodukte aber, die für die Wirkung ebenfalls in Betracht kommen, werden dabei überhaupt nicht berücksichtigt. $E_{s}$ bleiben eben auch hier nur wieder die Aufsehließverfahren. Relativ am besten wirkt das Verfahren, welches sich $A$. Falk hat patentieren lassen und dem wenigstens ein glücklicher Gedanke zugrunde liegt. Beim Fermen- 
tieren des Tabakblattes bildet sich, z. T. aus dem Nikotin, z. T. aus Eiweißstoffen, Ammoniak, welches im Blatte verbleibt. Das Nikotin selbst ist eine schwache Base, welche durch Ammoniak aus ihren Verbindungen ausgetrieben wird. Dieses Austreiben wird nach Falk durch Erhitzen bis zu Temperaturen von $195^{\circ}$ in fest geschlossenen Behältern bewirkt. Das Nikotin wird bei dieser Temperatur ausgetrieben, destilliert $a b$ und wird für sich aufgefangen. Ein praktischer Vorteil des Verfahrens besteht darin, daB die fertigen Zigarren, Zigaretten usw. der Entnikotinisierung unterzogen werden können. Die Tabakfabrikate werden ohne jede Vorbereitung, so wie sie sind, in Drahtkörben in den Heizraum eingesetzt; dieser ist so gut durch Isolierwände gegen Wärmeausstrahlung geschützt, daß die Temperatur äußerst langsam und in allen Teilen der Maschine sehr gleichmäBig steigt. Es destilliert neben Nikotin noch Ammoniak und Wasser heraus, während die wertvollen Bestandteile sich bei vorsichtiger Handhabung nicht wesentlich verringern. Nachdem der Apparat erkaltet ist, werden die Rauchrequisiten herausgenommen; sie sind natürlich strohtrocken geworden und müssen nun drei Tage auf dinenüberzogenen Gestellen in feuchten Kammern lagern, um dann völlig gebrauchsfähig zu sein und ihren normalen Feuchtigkeitsgehalt wieder zu gewinnen. Ich selbst habe oft solche Tabake analysiert und eine Nikotinverminderung von 30 bis $45 \%$ des ursprünglichen Gehaltes konstatieren können; Tatsache ist auch, daß gewiegte Raucher unbehandelte und in der beschriebenen Weise entnikotinisierte Zigarren usw, nicht unterscheiden konnten. Hier wird also das Aufschließen durch ein normalerweise bei der Erntebereitung im Blatte sich bildendes Produkt vorgenommen und der künstliche Eingriff besteht nur darin, daB man durch Erhöhung der Temperatur die Einwirkung desselben vervollständigt und gleichzeitig für eine Verflüchtigung des freigesetzten Alkaloids Sorge trägt. Ein anderer, vielleicht beachtenswerter Gedanke ist der, das AufschlieBen durch Kalkwasser vorzunehmen. Auch hier wird natürlich durch die Base das Nikotin in Freiheit gesetzt, gleichzeitig aber eine Auslaugung der organischen Säuren vermieden, denn diese (wie Oxalsäure, Apfelsäure usw.) geben mit Kalk unlösliche Verbindungen, die also dem Blatte und damit dem Rauchprodukte erhalten bleiben. Das freigewordene, leichtflüchtige Nikotin wäre dann unschwer zu entfernen. Allenfalls könnte man nun noch mit nikotinfreier Tabaklauge saucieren. Freilich, es sind noch andere Momente zu berücksichtigen. Durch die genannte Arbeitsweise dunkelt das Tabakblatt gewöhnlich stark nach und man muß dann noch für nachträgliches Bleichen Sorge tragen und was dergleichen kleine Schwierigkeiten mehr sind. Die Hauptsache aber, Entfernung eines großen Teiles des Nikotins bei erhaltenem Geschmack und Aroma könnte bei diesem Aufschließverfahren wohl erreicht werden und alles andere wäre Sache der technischen Durchbildung.

Wenn zum Schlusse noch die dritte Anwendung' des Aufschließens zu besprechen wäre, die Lockerung des Molekulargefüges bei Nahrungsmitteln zum
Zwecke der leichteren Zugänglichkeit für die Verdauungssäfte, so dürften hier einige Andeutungen genügen.

Dem Publikum am geläufigsten ist der Ausdruck ,aufgeschlossener Kakao". Der Holländer $C . J$ van Houten war der erste, welchem es gelang, dem Kakaopulver einen großen Teil seines natürlichen Fettgehaltes durch Abpressen zu nehmen, wobei dem zurückbleibenden Pulver seine nährenden Eigenschaften in vollem Maße gewahrt blieben. Durch diese Erfindung wurde die Tafelschokolade, die früher ausschließlich zux Herstellung der Trinkschokolade verwendet wurde, zum Teil aus dieser Verwendung verdrängt. Das Kakaopulver ist in Wasser oder in heiBer Milch nicht auflösbar, man mußte, wollte man die Nährstoffe des Kakaos möglichst ausnutzen und ihren Nährwert auch Kranken und Rekonvaleszenten zugänglich machen, nach möglichster Verteilung des Pulvers in der Flüssigkeit trachten, so daß also die Bildung eines Bodensatzes möglichst hintangehalten würde. Wenn man nun hydraulisch entfettetes Kakaopulver in heißes Wasser zu werfen versucht, wird man nur eine sehr geringe Verteilung, dagegen die Bildung eines sehr reichlichen Bodensatzes wahrnehmen. Soll der Kakao ,löslich" gemacht werden, so daß er sich in der heißen Flüssigkeit möglichst gleichmäßig verteilt, muß man danach trachten, gewisse Gewebeelemente des Kakaos, namentlich den Zellstoff durch chemische Eingriffe in eine Form zu bringen, in welcher sie sich in der Flüssigkeit fein verteilen und der Verarbeitung durch die Verdauungssäfte leicht zugänglich sind. Diese Operation nennt man das "Aufschließen" des Kakaos. Je weniger Bodensatz ein solcher aufgeschlossener Kakao erzeugt, je mehr sich in der Flüssigkeit schwebend erhält, desto wertvoller ist er. Eine vollkommene Löslichkeit wie beim Zucker oder Kochsalz ist beim Kakao ausgeschlossen; heute erzielt man dieses Aufschließen durch Behandeln des rohen oder des gerösteten Kakaos ohne oder nach Abpressen des Fettes mit kohlensaueren Alkalien, nämlich Pottasche, Soda, kohlensaurem Ammoniak. Durch die Alkalien wird der Zellstoff in einen gequollenen Zustand versetzt, in welchem er sich leichter verteilt, allerdings auch das Kakaorot, der Aromaträger, z. T. zerstört wird. Noch vor 25 Jahren wurde das Aufschließverfahren in Holland als tiefstes Geheimnis behandelt, heute ist es wohl Gemeingut der gesamten Industriewelt. Der halbgeröstete ind kleingebrochene Kakao wird mit $1 \frac{1}{2}-3$ Teilen Pottasche, in 20-30 Teilen aufgelöst, auf 100 Teile des entfetteten Präparates, bespritzt und damit imprägniert. Dann erst erfolgt das Fertigrösten und die übrigen beendenden Operationen; besonders wichtig ist hier ein weitgehendes Feinzerreiben, damit beim späteren, dem Entölen folgenden Vermahlen ein leicht siebbares Produkt erzielt werde, das beim Aufgießen mit Wasser möglichst wenig Bodensatz bildet. Ein Hauptnachteil des holländischen Verfahrens besteht darin, daB dem Kakao mit den nichtflüchtigen kohlensaueren Alkalien ein, wenn auch unschädlicher Fremdstoff zugeführt wird, der 
im Präparat verbleibt, wobei man allerdings bedenken mub, daß gerade Pottasche in der Asche aller Pflanzenstoffe in gröBerer oder geringerer Menge enthalten ist. übrigens arbeitet man in Deutschland vielfach mit dem flüchtigen kohlensauren Ammon, wobei der Kakao mit der Salzlösung in Mischmaschinen zusammengegeben und durch Einblasen von Dampf das beigefügte Wasser und das flüchtige Alkali, nachdem es das Aufschlieben besorgt hat, wieder entfernt wird. Der aufgeschlossenen, entölten und getrockneten Kakaomasse kann man schließlich wieder Fett in beliebiger Menge zusetzen, so daß es also auch möglich ist, aufgeschlossenen Kakao mit dem ursprünglichen Fettgehalt herzustellen. Mitunter wird auch blok mit Wasserdampf unter Druck, ganz ohne Zuhilfenahme von Alkalien aufgeschlossen, was aber weniger empfehlenswert ist, da die Stärke der Bohnen verkleistert, Säuregärung im Kakao eingeleitet und das fertige Endprodukt beim Lagern leichter von Schimmelpilzen befallen wird. Das mit fluchtigen Alkalien aufgeschlossene Produkt besitzt hellbraune Farbe, das unveränderte Kakaoaroma, reinen Geschmack ohne laugenhaften Beigeschmack, fühlt sich bei großer Feinheit ,wollig“" an und besitzt grofe ,Löslichkeit" in heiBen Flüssigkeiten. Alle holländischen Sorten, die durch nichtflüchtige Alkalien aufgeschlossen wurden, besitzen einen hohen - bis zu $8,19 \%$ - Aschengehalt; es wurde vielfach behauptet, daB durch diese Art der Behandlung der Kakao, namentlich durch die überreichliche Kalimenge, schwerer verdaulich gemacht wird. Daß dem nicht so sein kann, beweisen die ausgezeichneten, nicht nur in Holland, sondern auch in Deutschland nach holländischer Manier aufgeschlossenen Kakaosorten. Beim Genuß einer solchen Tasse Kakao aus $7,5 \mathrm{~g}$ Kakaopulver nimmt man nur $0,13 \mathrm{~g}$ Kali zu sich, während in einer Tasse Fleischbruthe $0,25 \mathrm{~g}$, in einem Glase Milch $0,31 \mathrm{~g}$, in einer Portion von $1 / 4 \mathrm{~kg}$ Kartoffelbrei sogar $0,61 \mathrm{~g}$ Kali enthalten sind. Tatsächlich haben Versuche mit aufgeschlossenem Kakao ergeben, daß davon $90 \%$ verdaut werden, wobei gerade die Aschenbestandteile vollkommen in den Körper übergehen.

Allbekannt sind die diesbezüglichen Veränderungen, welche alle Mehlprodukte beim Backen erfahren. Die Stärkekörner sind gequollen, ihre Zellhäute gesprengt, sie sind verkleistert, werden beim Backen z. T. abgebaut, zu Dextrinen und sogar bis zu Zucker zerlegt. Durch das Anmachen des Teiges und das Backen findet also eine Aufschließung des Stärkekornes statt. Bei der Herstellung von Findermehlen u. dergl. benutzt man zur vorherigen Aufschließung das Enzym Diastase, welche bekanntlich im keimenden Samenkorn die Verwandlung der unlöslichen Stärke in lösliche Kohlehydrate, Dextrine und Malzzucker, bewirkt. Auch bei der Fabrikation löslicher Eiweibnahrung sind Fortschritte erzielt worden, der Nährwert des Fleisches kann heute sogar ohne jede Arbeit für die verdauenden Enzyme dem Organismus einverleibt werden, nachdem dessen Eiweiß künstlich vollkommen abgebaut worden ist. Alle diese mehr oder weniger lösliches Eiweiß enthaltenden Präparate kommen unter den versehiedensten Namen auf den
Markt kranken aber freilich alle an dem Mangel jeden Geschmacks, wenn sie nicht gar unangenehm schmecken, denn die Albumosen und Peptone genannten Abbauprodukte von natürichem Eiweil schmecken bitter. Außer Stärke und Eiweiß gibt es aber noch andere hoch zusammengesetzte, schwer verdauliche Nahrungsstoffe, wie das Inulin, deren Aufschließen wichtige Nährquellen eröffnen könnte. Jedenfalls ist die Technik der Aufsehließung nicht minder für die Gewinnung wie für die Entfernung von Nahrungs- und GenuBstoffen von allergröbter Wichtigkeit.

\section{Besprechungen.}

\section{Neuere Literatur über Photographie.}

1) Eder, Josef Waria, Austuhriches Handbueh der Fhotographie. Band I, Teil I-IV. Dritte, gänzlich umgearbeitete und vermehrte Auflage. Halle 1905 bis 1912. Wilhelm Knapp. Preis komplett 67 Mark

Der große Kreis derjenigen, welche die Photographie ernstlich betreiben, sei es als Selbstzweck mit künstlerischen Zielen, sei es als Tilfsmittel zu wissenschaftiichen Zwecken, wird es mit großer Freude begrußen, daß der erste Band des Ausfïhrlichen Handbuchs der PhotoGraphie von Josef Maria Eder mit dem Erscheinen des dritten Teiles nummehr in dritter, gänzlich umgearbeiteter und vermehrter Auflage fast vollstindig vorliegt. Ist doeh waer der berufenste Fachmann fur die Schatfung eines grob angelegten Werkes, das das Riesengebiet der Lichtbildkunst mit allen ihren Hilfswissenschaften erschöpfend behandelt. In der Tat ist der erste Band für alle diejenigen Gebiete, die er umfaßt, wieder zu einem niemals versagenden Nachschlagewerk und Lehrbuch allerersten Ranges geworden, das alle modernen Errungensichaften der rastlos sich vervollkommnenden und damit fur immer weitere Kreise anderer Wissen schaften unentbehrlichen Photographie unfaBt. Nur wer alle die in den letzten Jahren neu geschaffenen Anwendungsmöglichkeiten der Tichtbildnerei aufmerksam verfolgt hat, wird ermessen können, welche Riesenarbeit der hochverdiente Verfasser in diesem umfassenden Tandbuch niedergelegt hat.

T. Teil. Geschichte der Photographie. (484 und XVI Seiten. Gr. $8^{0}$ mit 148 Abbildungen und 12 Tafeln. Preis 12 Mark.) Eder hat in diesem stattlichen Bande seine fruher publizierten grundlegenden und aut eingehendem Quellenstudium beruhenden geschichtichen Abhandlungen bis in die neueste 7 eit hinein vervoll stiundigt und schildert hier zum ersten Male die gesamte Friindungsgeschichte der Photographie bis zum Ende des 19. Jahrhunderts. Einen besonderen Reiz erhált das Werk dadurch, das ihm in vorzüglicher Wiedergabe eino große Anzahl von zum Teil äußerst seltenen und sehwer zugänglichen Inkunabeln und Bildnissen beigegeben ist, welche auf die Geschichte der Photographie Bezug haben. Das ganze Buch ist fesselnd und mit gröbter Objektivitat geschrieben. Ausgehend vou den sich im Altertum beLampfonden Ansichten des Plato und des Aristoteles über das Sehen und der schon dem Vitruvius bekannten Hinwirkung des Sonnenlichts auf gewisse Stoffe kommt der Verfasser über die verworrenen Ansichten der Alchimisten über den EinfluB des Sonnenlichts kurz hinweggehend zur ersten Beobachtung eines chemischen. Sehwärzungsprozesses durch Albertus Magnus und damit zu den eigentlichen Uranfängen der Photographie. Ex schildert 